\title{
Detection of Methicillin Resistant Staphylococcus aureus (MRSA) in Surgical Site Infections in a Tertiary Care Hospital
}

\author{
V. Aruna and S. Rajesh* \\ Department of Microbiology, Government Mohan Kumaramangalam \\ Medical College, Salem, Tamil Nadu, India \\ *Corresponding author
}

\author{
A B S T R A C T
}

\begin{tabular}{|l|}
\hline Ke y w o r d s \\
Cefoxitin, Surgical \\
site infection, \\
MRSA.
\end{tabular}

MRSA (Methicillin resistant Staphylococcus aureus) is especially commonly found in post-operated patient. These MRSA strains, showing resistance to many of the antibiotics are becoming more difficult to treat and thus prove to be dangerous. The aim of the study was to screen for MRSA in surgical site infections by disc diffusion method using cefoxitin, from which surgical department the patients were more affected by these MRSA strains and to find the precipitating factors for the development of MRSA strains. Sixty six Staphylococcus aureus were isolated from 124 pus, wound swab samples collected in various surgical departments and processed in microbiology department of, Government Mohan Kumaramangalam medical college hospital. They were confirmed by microscopy, culture and biochemical reactions. MRSA were detected by disc diffusion test using cefoxitin $(30 \mu \mathrm{g})$ disc. Majority of Staphylococcus aureus were isolated from pus samples from surgery department (48\%). Least number of MRSA was encountered in obstetrics and gynaecology department (4\%). Detection of MRSA is of utmost importance for treating patients and to prevent mortality.

\section{Introduction}

Infections have been one of the major causes of morbidity and mortality worldwide among human population ${ }^{(1)}$. Staphylococcus aureus, one of the earliest detected bacteria was discovered by Sir Alexander Ogston in 1880 in Aberdeen, Scotland ${ }^{(2)}$. S. aureus is responsible for a wide range of infections most notable among which are Neonatal sepsis $^{(3)}$, Endocarditis ${ }^{(4)}$, Bacteremia ${ }^{(5)}$, and Skin infections. S. aureus is an important pathogen causing serious infections both in hospitals and Community. These resistant microbial strains have become one of the major concerns of the Clinicians, Microbiologists and Public Health officials.
MRSA is especially troublesome in hospitals, where patients with open wounds, invasive devices and weakened immune systems are at greater risk of nosocomial infection. Resistance to antimicrobial agents developed by microorganisms has created a major problem in the treatment of not only serious and life threatening infections in Hospitals but also common infections at the Community level. Those patients who are in the intensive care units and burns wards and infants and elderly are particularly vulnerable ${ }^{(7)}$. Cefoxitin $\operatorname{discs}^{(6)}$ is used to find out the susceptibility of S.aureus to Methcillin by Kirby Bauer method. ${ }^{(17,18)}$ Since Methicillin is 
an unstable compound either Oxacillin or Cefoxitin discs may be used to screen MRSA. Cefoxitin is the potent inducer of mecA gene ${ }^{(8)}$ and hence Cefoxitin is the most reliable to detect MRSA by this method

\section{Materials and Methods}

The present study was conducted in Government Mohan Kumaramangalam Medical College Hospital, Salem. The study period was for 2 months from the period august 2015 to September 2015. Samples were received from in patients hospitalized from various surgical field at Government Mohan Kumaramangalam Medical College Hospital. Staphylococcus aureus isolates from clinical samples including, pus, and wound swab were included in the study which were collected from infected patients after the surgical procedure from various surgical sites to screen for MRSA. Consent is obtained from the patient before the collection of sample. For the collection of sample, a sterile swab is used and is gently rotated on the area to collect exudate from the wound and is placed into the transport medium. In the case of pus, the pus is collected in a sterile container and is sent to the laboratory.

The samples were properly labelled with name, age, sex and IP/OP number of the patient, date and time of collection of the sample and processed immediately. All the above specimens were inoculated on to the nutrient agar, blood agar, and MacConkey agar, and incubated at $37^{\circ} \mathrm{C}$ for $18-24$ hours aerobically and observed after incubation. All the suspected colonies were identified by colony morphology, white opaque colonies in blood agar, tiny pink colonies in MacConkey agar and golden yellow colonies in nutrient agar and gram staining was done. Various biochemical reactions are done. They are catalase positive, oxidase negative, MR and VP positive and indole negative. Further confirmation was done by slide and tube coagulase test. The sensitivity to antibiotics was done by Kirby Bauer Disc Diffusion method as recommended by CLSI. Control strains used were Staphylococcus aureus ATCC -25923 and MRSA-ATCC 43300.The media used in Kirby Bauer testing is Mueller-Hinton agar. Pure inocula was obtained by selecting 4-5 colonies from the culture plates and inoculating them into broth medium (peptone water) to match the turbidity of 0.5 McFarland standard, and incubated for 3-5 hours. Using a swab, it is streaked over Mueller-Hinton agar. For uniform growth, it is streaked in perpendicular direction over the agar. An antibiotic disk dispenser is then used to place the antibiotic disc 30 microgram of Cefoxitin in Muellar Hinton agar medium inoculated with $S$. aureus and the zone of inhibition is observed after 24 hour incubation at $35^{\circ} \mathrm{C}$. In cefoxitin disc diffusion test zone diameter of $22 \mathrm{~mm}$ or more was taken as sensitive and $21 \mathrm{~mm}$ or less was considered as resistant. These resistant isolates were considered as MRSA.

\section{Results and Discussion}

The present study was conducted in Government Mohan Kumaramangalam Medical College Hospital, Salem. The study period was for 2 months from the period august 2015 to September 2015. A total of 124 pus and wound swab samples were collected over a period of 2 months from various surgical departments. Out of which 55 samples were collected from the General Surgery department, 26 samples from the Orthopedics department, 25 samples from the Plastic Surgery department, 7 samples from the Obstetrics and Gynecology department and 11 samples from the Pediatric Surgery department.

Out of these 124 samples, 66 samples showed positive for Staphylococcus aureus. 32 samples from Surgery, 15 samples from 
Orthopedics, 12 samples from Plastic Surgery, 3 samples from O\&G and 5 samples from Pediatric Surgery department were positive for Staphylococcus aureus.

By cefoxitin disk diffusion method, MRSA samples were detected. A total of 28 samples were positive for MRSA and 38 MSSA (Table 1) 14 samples from Surgery, 6 samples from Orthopedics, 5 samples from Plastic Surgery, 1 sample from O\&G and 2 samples from Pediatric Surgery department were positive for MRSA (Table 2).

Of the patients tested positive for MRSA, $82.1 \%$ of the patients were hospitalized for more than 5 days ( 23 out of 28 patients). 25 of the 28 patients were given antibiotic cefotoxime after their operative procedure. It was observed that MRSA was tested positive after the patients had undergone minor procedures like draining of abscess from the affected area. 9 patients were tested positive for MRSA when the sample was collected a day after the abscess removal.5 patients were tested positive for MRSA after wound debridement procedure. 3 patients were tested positive for MRSA after an open surgery for fracture femur (2), radius (1) was done (fixation of a comminuted fractured using Kirschner wire) and 3 cases operated for osteomyelitis in orthopaedic ward.

In the plastic surgery ward, 5 patients were tested positive for MRSA after collagen sheet was applied over the area of burns. In Pediatric Surgery department 2 samples of wound infection showed positive for MRSA. One patient from O\&G department showed positive for MRSA after LSCS was done. It is noted that the patient was hospitalized for 13 days.

MRSA was reported high in surgery department $(50 \%)$. The low incidence in obstetrics and gynaecology department (4\%) may be due to minimal number of hospital stay and clean wound. The increase in the incidence of infections due to $\mathrm{S}$. aureus is partially a consequence of advances in patient care and also of the pathogen's ability to adapt to a changing environment.

Image.1 MRSA Detection Using Cefoxitin Disc

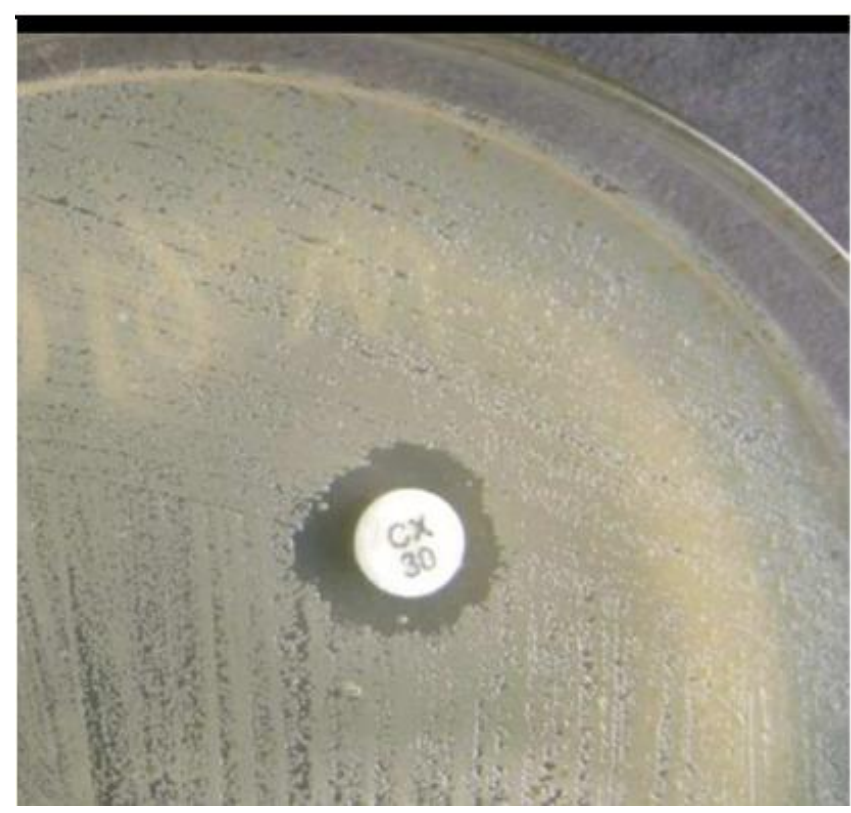


Table.1 prevalence of MRSA among Staphylococcus aureus isolates

\begin{tabular}{|l|l|l|}
\hline $\begin{array}{l}\text { No. of Staphylococcus aureus } \\
\text { isolates }\end{array}$ & MSSA & MRSA \\
\hline 66 & $38(58 \%)$ & $28(42 \%)$ \\
\hline
\end{tabular}

Table.2 Distribution of MRSA in wards- $\mathrm{n}=66$

\begin{tabular}{|l|c|c|}
\hline Ward & Staphylococcus aureus (66) & MRSA(28) \\
\hline Surgery & $32(48 \%)$ & $14(50 \%)$ \\
\hline Orthopaedics & $15(22 \%)$ & $6(21 \%)$ \\
\hline Plastic surgery & $12(18 \%)$ & $5(18 \%)$ \\
\hline $\begin{array}{l}\text { Obstetrics } \\
\text { Gynaecology }\end{array}$ & $3(4 \%)$ & $1(4 \%)$ \\
\hline Paediatrics & $5(7 \%)$ & $2(7 \%)$ \\
\hline
\end{tabular}

Van Beikum et al., ${ }^{(9)}$ explained that the incidence of MRSA was steadily increasing since its emergence in 1960s. The increased trend may be due to the adverse use of antibiotics resulting in faulty genetic background or poor infection control practices in the environment.

In this study, $42 \%$ of MRSA was isolated from pus samples and this is supported by the study of Trivete and Kirkland ${ }^{(10)}$ (1999) which showed $30 \%$ MRSA in pus samples. The increased incidence of MRSA in wound infection may be due to the production of Panton Valentine Leucocidin (PVL) by MRSA which is known to be associated with tissue necrosis. Rajaduraipandi et al., ${ }^{(1)}$ (2001) documented $37.9 \%$ MRSA and on contrary Anuparba et al., ${ }^{(1)}$ (2006) documented 54.8\%.

Tacconelle et al., ${ }^{(13)}$ (2008) proved the association of prolonged exposure to quinolone antibiotic with MRSA infection. The occurrence of MRSA in various infections with associated risk factors may be due to acquired chemotactic defects or opsonic defects. The text book of Microbiology by Baveja ${ }^{(14)}$ also explains the occurrence of $S$. aureus as prime organism in all infections and states that this is due to the presence of a number of enzymes and toxins produced by $S$. aureus which may inhibit phagocytic and opsonic mechanisms. Presence of predisposing factors such as prolonged hospital stay, antibiotic intake may be the reason for high MRSA report among inpatients ${ }^{(15,16)}$. This might be attributed to prolonged hospital stay in cases of fractures and operative procedures. This is the reason why higher rate of MRSA carriage has been reported in the Surgical, Orthopaedic, Obstetric and gynecological wards. Patients with extensive skin lesions are heavy shedders of MRSA. So the rate was high in plastic surgery ward. It was due to big surface area of denuded skin with large inoculam of organism that can easily be transmitted to other patients via hands of health care workers.

The common complications following all operative procedure is surgical site infections. Preoperative care, the theatre sterility, postoperative care, overcrowding, and the type of surgery are some of the factors which determine the surgical site infections. Contamination from the external environment is the most probable reason for the wound infection. This study was focused on finding out of a simple, economic and more accessible method to identify MRSA which is resistant to many antibiotics and it is very difficult to eradicate from patients as well as carriers. The therapeutic options are limited and the spectrum 
of resistance is worrying. The organism has an ability to spread. MRSA is a threat not only to immunocompromised individuals, but also to general public. Detection of MRSA is of utmost importance for treating patients and to prevent its spread.

\section{References}

1. Harrison's Text Book of Principles of Internal medicine. $17^{\text {th }}$ Edition. Pp. 749.

2. Curran, JP, Al.Salihi. FL. Neonatal Staphylococcal skin and soft tissues infection: massive outbreak due to an unusual phage type. Peadiatrics. 1980; 66: 285-90.

3. Melish and Glasgow. Staphylococcal scalded skin syndrome, the expanded clinical syndrome. J. Pediatrics, 1971; 78:958-62.

4. Wentland. M.P, George. Y. Lesher, Hooper. D.C. American Society for Microbiology 1993; XIII-XIV.

5. Fowler. V.G, Oslen. M.K, Corey. G.R. et $a l$, Clinical identifiers of complicated $S$. aureus bacteremia. Arch.Intern.Med. 2003; 163:2066-72.

6. Fernandes. C.J, Colligan. P et al., Cefoxitin resistance as a surrogate marker of MRSA. J. Clinic. Microbiol. 2005; 55: 506-10.

7. Cosgrove. S.S, Sakoulas. G, Casmell. Y. Comparison of mortality associated with MRSA and MSSA, a meta analysis.Clinic.Infect.Dis. 2003; 36: 53-59.

8. Swenson. J.M, Williams. P.P, Killgor. G, Mohr. C et al., Performance of 8 methods including 2 new rapid methods for detection of MRSA. J.Clinic.Microbiol. 2001. 39: 3785-88.

9. Van Beikam. A, Verbrug, $\mathrm{H}$ et al., 40 years of MRSA. B.M.J 2001; 323: 644-45.

10. Trivette.SL, Kirkland.KB, Briggs.JP et al., Impact of Surgical site infection; attributable mortality and excess length of Hospitalisation Infect. control. Hosp. Epidemiol. 1999; 20:725-30

11. Rajadurai Pandi. K, Mani. K.R, Panneerselvam. K, Bhaskar. $\mathrm{M}$ et al., Prevalence and microbial susceptibility pattern of MRSA - a multicentric study. IJMM, 2006; 24: 34-38.

12. Anupurba. S, Sen. MR, Nath. G, Sharma. BM. Prevalance of MRSA in a tertiary care hospital in Eastern Uttar Pradesh. I J M M 2003; 21: 49-51.

13. Tacconelli. E, De. Angelis. G, Catalado. M.A et al., Does Antibiotic exposure increase the risk of MRSA isolation? A systematic review and meta analysis. J. of Antimicrob. Chemother. 2001; 48: 143-44.

14. Baveja's Text book of Microbiology. $3^{\text {rd }}$ edn, Pp: 177.

15. Fomda BA, Thokar MA, Khan A, Bhat JA, Zahoor D, Bashir G, et al., Nasal carriage of Methicillin resistant Staphylococcus aureus among healthy population of Kashmir, India. Indian J Med Microbiology 2014; 32:39-43

16. Klein E, Smith DL, Laxminarayan R. Hospitalizations and deaths caused by methicillin-resistant Staphylococcus aureus, United States, 1999-2005. Emerg Infect Dis 2007;13:1840-6

17. Drew WL, Barry AL, O'Toole R, Sherris JC. Reliability of the Kirby-Bauer Disk diffusion method for detecting Methicillinresistant strains of Staphylococcus aureus. Appl.Microbiol., 1972; 24:240-7

18. Boutiba-Ben Boubarker I, Ben Abbes R, Ben Abdalklah H, Mamlouk, et al., Evaluation of cefoxitin disk diffusion test for the routine detection of methicillinresistant Staphylococcus aureus. Clinical Microbiol. Infect., 2014; 10:762-5.

\section{How to cite this article:}

Aruna, V. and Rajesh, S. 2017. Detection of Methicillin Resistant Staphylococcus aureus (MRSA) in Surgical Site Infections in a Tertiary Care Hospital. Int.J.Curr.Microbiol.App.Sci. 6(12): 42044208. doi: https://doi.org/10.20546/ijcmas.2017.612.483 